\title{
BMJ Open PC6 acupoint stimulation for the prevention of postcardiac surgery nausea and vomiting: a protocol for a two-group, parallel, superiority randomised clinical trial
}

\author{
Marie Cooke, ${ }^{1}$ Claire Rickard, ${ }^{1}$ Ivan Rapchuk, ${ }^{2}$ Kiran Shekar, ${ }^{3}$ Andrea P Marshall, ${ }^{4}$ \\ Tracy Comans, ${ }^{5}$ Suhail Doi, ${ }^{6}$ John McDonald, ${ }^{7}$ Amy Spooner ${ }^{8}$
}

To cite: Cooke M, Rickard C, Rapchuk I, et al. PC6 acupoint stimulation for the prevention of postcardiac surgery nausea and vomiting: a protocol for a two-group, parallel, superiority randomised clinical trial. BMJ Open 2014;4:e006179. doi:10.1136/bmjopen-2014006179

- Prepublication for this paper is available online. To view these files please visit the journal online (http://dx.doi.org/10.1136/ bmjopen-2014-006179)

Received 22 July 2014 Revised 10 October 2014 Accepted 17 October 2014

CrossMark

For numbered affiliations see end of article.

Correspondence to Professor Marie Cooke; m.cooke@griffith.edu.au

\section{ABSTRACT}

Introduction: Postoperative nausea and vomiting (PONV) are frequent but unwanted complications for patients following anaesthesia and cardiac surgery, affecting at least a third of patients, despite pharmacological treatment. The primary aim of the proposed research is to test the efficacy of PC6 acupoint stimulation versus placebo for reducing PONV in cardiac surgery patients. In conjunction with this we aim to develop an understanding of intervention fidelity and factors that support, or impede, the use of PC6 acupoint stimulation, a knowledge translation approach.

Methods and analysis: 712 postcardiac surgery participants will be recruited to take part in a two-group, parallel, superiority, randomised controlled trial. Participants will be randomised to receive a wrist band on each wrist providing acupressure to $\mathrm{PC}$ six using acupoint stimulation or a placebo. Randomisation will be computer generated, use randomly varied block sizes, and be concealed prior to the enrolment of each patient. The wristbands will remain in place for $36 \mathrm{~h}$. PONV will be evaluated by the assessment of both nausea and vomiting, use of rescue antiemetics, quality of recovery and cost. Patient satisfaction with PONV care will be measured and clinical staff interviewed about the clinical use, feasibility, acceptability and challenges of using acupressure wristbands for PONV.

Ethics and dissemination: Ethics approval will be sought from appropriate Human Research Ethics Committee/s before start of the study. A systematic review of the use of wrist acupressure for PC6 acupoint stimulation reported minor side effects only. Study progress will be reviewed by a Data Safety Monitoring Committee (DSMC) for nausea and vomiting outcomes at $\mathrm{n}=350$. Dissemination of results will include conference presentations at national and international scientific meetings and publications in peer-reviewed journals. Study participants will receive a one-page lay-summary of results. Trial registration number: Australian New Zealand Clinical Trials Registry—ACTRN12614000589684.

\section{INTRODUCTION}

Postoperative nausea and vomiting (PONV) are common unwanted complications for patients following anaesthesia/cardiac surgery, affecting at least one in three patients, despite pharmacological prophylaxis and/or treatment. A Cochrane Systematic Review (CSR) specific to medicines for preventing PONV, concluded that PONV affects around 80 of every 100 individuals undergoing surgery, and that if all 100 were given a drug to prevent PONV, only around 28 would benefit. ${ }^{1}$ The burden of caring for patients postcardiac surgery is immense, with the Australian Institute of Health and Welfare (AIHW) ${ }^{2}$ annual report indicating that in Australia alone nearly 179000 procedures involving the cardiovascular system were performed between 2011 and 2012. Cardiovascular disease (CVD) remains the most expensive diagnostic group to treat in Australia, costing about \$A7.9 billion in 2008-2009, with over half of this spent on patients while admitted to hospital. ${ }^{3}$ Similarly, the significant cost of CVD to the UK healthcare system in 2009 was reported to be around $£ 8.6$ billion with $50 \%$ of this attributed to hospital care. ${ }^{4}$

As part of their treatment and recovery, cardiac surgery patients experience varying rates of PONV. Studies in the 1990s found rates of PONV in cardiac surgery patients of $22 \%{ }^{5} 47 \%{ }^{6}$ and $50 \% .^{7}$ More recent studies reported rates of: $39-42 \%$ in a North American randomised controlled trial (RCT) $;^{8}$ $26-27 \%$ in a systematic review of 10 RCTs; ${ }^{9}$ and $35 \%$ in a Canadian study. ${ }^{10}$ Patients report that they have a strong preference for avoiding PONV $^{11}$ and, of 10 negative outcomes of surgery, rank vomiting as the most undesirable outcome and nausea as the fourth most undesirable. ${ }^{12}$ Patient dissatisfaction with anaesthetic care is strongly related to PONV. ${ }^{13}$ PONV can delay transfer from the recovery unit by up to $20 \mathrm{~min}^{12}$ and vomiting can place 
tension on sutures and wounds, produce imbalances in body electrolytes, and cause bleeding. ${ }^{12}$ Acupressure is a therapeutic intervention endorsed by the $\mathrm{WHO}^{14}$ and an alternative approach thought to prevent nausea and vomiting through an alteration in endorphins and serotonin levels.

\section{Efficacy of acupressure for PONV}

Acupressure as a traditional Chinese medicine has been practised for centuries. The concept is based on life energy (Qi) flowing through channels known as meridians through the body. ${ }^{15}$ It is argued that acupressure restores equilibrium to disruptions affecting the body's homeostasis by stimulating specific points (acupoints) that connect the meridians to organs. ${ }^{15}$ Although the mechanism for the action of acupressure has not been scientifically investigated fully, it is thought that it may prevent nausea and vomiting through an alteration in endorphins and serotonin levels. ${ }^{16}$ PC6 point stimulation for treating nausea and vomiting was reported in the early 1990s. ${ }^{17}$ The WHO (Western Pacific Regional Office) reached consensus on acupuncture point locations and published guidelines in $2008 .^{18}$ The PC6 acupoint is the meridian point in the pericardium channel, and is located on the inner forearm between the extensor carpi radialis and palmaris longus tendons, one-sixth of the distance from PC7 on the medial wrist crease to PC3 in the cubital fossa. ${ }^{18}$ Measuring the distance between the palmar wrist crease and inner forearm with a tape measure, and placing the bead on the wristband between the two tendons a sixth of the distance measured, is quick, acceptable and feasible in the clinical environment. This method is much more accurate than the previously used procedure of using the three middle fingers on the inside of the patient's wrist to measure distance. Although the PC6 acupoint can be stimulated with a variety of methods (acu-stimulation device, acupressure, acupuncture, capsicum plaster), the important concept is stimulation of the correct acupoint. ${ }^{19} \mathrm{~A}$ meta-analysis in a recent Cochrane Systematic Review (CSR) by Lee and Fan $^{19}$ of 40 trials totalling 4858 participants (all surgical patients without age limits), reports a clear positive effect of PC6 acupoint simulation on: nausea (RR $0.71,95 \%$ CI 0.61 to 0.83 ); vomiting (RR $0.70,95 \%$ CI 0.59 to 0.83 ); and need for rescue antiemetics (RR $0.69,95 \%$ CI 0.57 to 0.83 ). Given the extensive use of PC6 acupoint in reported research studies in relation to $\mathrm{PONV}$ in the literature and its ease of use in clinical practice, this acupoint has been chosen for this research.

Although the Lee and Fan ${ }^{19}$ meta-analysis identified a clear positive effect of PC6 acupoint stimulation, the analysed studies were conducted in various clinical settings and with different populations, suggesting that, 'on average', the intervention is known to be effective. It is thought that this intervention is not used in clinical practice despite the positive CSR and the reasons for this are unknown but perhaps related to the following factors. The CSR meta-analysis incorporated only one study undertaken in a cardiac population $(n=152)$, and included various methods of PC6 acupoint stimulation versus sham/drug therapy for prevention of PONV. The vast majority of studies had small sample sizes (range 36-250), with only one with a reasonable sample size $\mathrm{n}=410$ (sample size calculated on the CSR meta-analysis outlined below indicates a sample of $>700$ is required); quality of the studies is highly varied, with concerns mostly regarding allocation sequence generation and allocation concealment, which this proposed study addresses. As such, it is argued that (1) a large rigorous RCT is needed to provide definitive evidence to inform clinicians and (2) the direct application of this to cardiac population needs further consideration and investigation. There is also the added significant value in the current planned study of incorporating secondary hypotheses around dose-response (dose varied considerably across studies in the CSR) and quality of recovery (rarely addressed in CSR studies) to yield new knowledge and draw conclusions for postoperative management and patient care. The economic evaluation including the side effects associated with drugs used to treat PONV (eg, for two common antiemetics: (1) Metoclopramide's side effects include sedation and dystonic reactions and (2) Ondansetron's side effects include headache, dizziness and possible QT interval prolongation) will also provide guidance on the value for money offered by this intervention. Further, despite the CSR, use of acupressure for PONV is not widely practiced, and methods will be used in this study to develop a comprehensive understanding of factors relevant to the successful implementation of acupressure for PONV, a strategy that is recommended when there is a degree of uncertainty about an intervention. ${ }^{20}$ These data will help us to understand factors which might impede implementation, and allow for targeted implementation strategies to be developed, should the study results demonstrate a positive impact. ${ }^{21}$

This two-group, parallel, superiority, blinded, RCT will test the efficacy of PC6 acupoint stimulation versus placebo for reducing PONV in cardiac surgery patients. Primarily this study aims to investigate whether patients in the PC6 acupoint stimulation group will experience significantly less nausea and vomiting in the first $36 \mathrm{~h}$ following admission to intensive care unit (ICU) postcardiac surgery, than patients in the sham group. It also aims to investigate whether (1) Patients in the PC6 acupoint stimulation group will experience: (1) significantly less severe nausea postoperatively than patients in the sham group in the first $36 \mathrm{~h}$ postoperatively; (2) significantly less early-onset $(\leq 16 \mathrm{~h})$ and late-onset $(>16 \mathrm{~h})$ PONV than patients in the sham group; (3) a greater reduction in rescue drug therapy postoperatively than patients in the sham group in the first $36 \mathrm{~h}$ postoperation; and (4) a greater quality of recovery at morning of day 4 than patients in the sham group. (2) Costs associated with treatment for PONV will be significantly 
lower in the group using PC6 acupoint stimulation than in the sham group. Previous PC6 acupoint stimulation studies for PONV have mostly used durations of 6,12 and $24 \mathrm{~h}$. The duration of acupoint stimulation chosen for this study is $36 \mathrm{~h}$, as this will take account of postcardiac surgery patients who may be intubated and ventilated for 2-6 h after surgery. The $36 \mathrm{~h}$ instead of 24 would ensure that we have a full $24 \mathrm{~h}$ period with patient awake/extubated and mobilising. A parallel aim is to use an integrated knowledge translation approach to develop a comprehensive understanding of factors that impact on successful implementation of the intervention. The focus will be on the delivery of the intervention as intended, processes of implementation and change, and responses of patients and healthcare professionals to the intervention. Patients' satisfaction with their PONV care will be measured and clinical staff interviewed about the clinical use, feasibility, acceptability and challenges of using acupressure wristbands for PONV in practice. These data can then be used to assist implementation should the intervention be shown to be effective. If effective, this intervention has the potential to significantly improve the quality of care for hundreds of thousands of patients worldwide, each year through a cost-effective and safe intervention for the prevention and management of PONV.

\section{METHODS AND ANALYSIS \\ Study design}

The study will use a two-group, parallel, superiority, participant and clinician-masked RCT design. Participants will be postoperative adult cardiac surgery patients. The intervention will be PC6 acupoint stimulation. The main outcome measure will be PONV, with secondary outcome assessment of severe nausea, difference in effect early-onset and late-onset postoperatively, need for rescue antiemetic therapy and quality of recovery by fourth postoperative day. An economic substudy will compare costs associated with PC6 stimulation device, costs of antiemetic medication, and hospital length of stay in the two groups. Also incorporated will be a parallel integrated knowledge translation approach, to develop understanding of intervention fidelity and factors that support, or impede, the use of PC6 acupoint stimulation. A superiority design has been chosen as this is consistent with the literature to date: the CSR of 40 trials found that all except one trial indicated less nausea in the group receiving PC6 stimulation compared to control. In addition there is no biologically plausible reason that PC6 acupressure would increase PONV. Use of a sham will eliminate the influence of treatment effects other than those caused by the treatment itself (ie, knowledge of receiving the treatment and expectations of what it might do etc.) by blinding participants, clinicians and also members of the research team, as to who is receiving the acupressure and who is not. The CONSORT guidelines ${ }^{22}$ with its official extention of Standards for Reporting Interventions in Clinical Trials of Acupuncture $)^{23}$ for reporting trials have been used to guide study design.

\section{Setting and population}

Trial participants will be sampled from an adult postcardiac surgery population. This population reflects a relatively homogenous group and is, thus, likely to detect an effect if one exists in the population. Two hospital sites will be accessed where on average 22 patients undergo cardiac operative procedures consistent with the inclusion criteria each week. Only patients undergoing primary surgical procedures will be included as patients undergoing second or subsequent cardiac surgery are more likely to have variable preoperative, intraoperative and postoperative course and care, and the standardised protocol outlined below in concurrent treatment may not be applicable. It is anticipated that recruitment will take 18 months.

\section{Sample size}

The primary outcome of nausea was used to power this study. Only one previous study has specifically examined the effect of acupressure on nausea with a cardiac postoperative group, finding that the proportion of participants with observed nausea in the control group was $35 \%{ }^{10}$ which is consistent with our unpublished preliminary data. Based on the CSR, to detect a $30 \%$ reduction in relative risk of nausea ${ }^{19}$ with $90 \%$ power, a total of 712 participants ( $\mathrm{n}=356$ per group) at an $\alpha$ of 0.05 (for a superiority test of two independent proportions) ${ }^{24}$ is needed.

\section{Recruitment}

A Registered Research Nurse (RRN) will identify elective patients from operation lists and approach each patient (at preadmission clinic or on ward) to introduce the study. Those interested will then be formally screened and those eligible will be provided with an information sheet, further explanation of the study and clarification of any questions with the contact details of the study manager provided as a contact for further information. Written informed consent will be obtained. Patients, who meet all of the inclusion criteria, and none of the exclusion criteria, will be eligible. The inclusion criteria are: elective or urgent primary cardiac surgery (coronary artery bypass Graft (CABG); valve and double valve replacement; CABG plus single valve replacement); able to understand, speak, read and write English or have a suitable interpreter available; aged 18 years or over, and able to give informed consent. Exclusion criteria are: impaired renal function-creatinine level $>200$ or estimated-glomerular filtration rate $<40$; patients receiving: antiemetic medication within $24 \mathrm{~h}$ prior to surgery, or histamine H2-receptor antagonist within $24 \mathrm{~h}$ prior to surgery; skin damage (eg, burn scars) over PC6 area; wrist circumference $>21 \mathrm{~cm}$; and any previous experience of acupressure for nausea and/or vomiting, for 
example, related to morning sickness, chemotherapy or travel/motion sickness.

\section{Randomisation and allocation concealment}

Computer-generated random assignment will occur at the point of study entry, and each patient will be allocated to a numbered trial group. Randomisation will involve a 1:1 ratio; stratified assignment by risk of nausea (Apfel Score that can be stratified into low (score 0 or 1 ), moderate (score 2), extremely high (score 3 or 4$)^{25}$ at study site, with random variation in block sizes of 4-10. RRN will obtain a participant code number corresponding to a study pack to which each participant will be randomly allocated using a web-based independent automated service at the university Clinical Trials Randomisation Service, which is overseen by a biostatistician, and record the study group code in the patient's medical record and on study forms. This process ensures adequate concealment, limiting likelihood of selection bias. ${ }^{26}$

\section{Processes to ensure blinding}

RRN will obtain the participant code number and, thus, is blinded to group allocation and will collect outcome data on day 4 postoperatively and document final nausea scale score at $36 \mathrm{~h}$. Another RRN (RRN2, trained and assessed to ensure correct PC6 positioning) will apply the intervention/placebo on arrival in the ICU following surgery. All clinicians providing care will be blinded to group allocation. ICU and ward registered nurses will collect nausea scores and incidence of vomiting. All patients will be blinded to group allocation, as an occlusive bandage will be applied over the wristband. The acupressure wristband will be identical in appearance and position for both intervention and placebo groups. All members of the research team involved in participant recruitment, randomisation and data collection, will be blinded to group allocation.

\section{Intervention}

Participants in the acupressure group will have a Seaband wristband applied on arrival to ICU on both wrists (bilateral application is recommended) by RRN2 ensuring that the bead stimulates the PC6 acupoint and the bands are covered with a light opaque bandage. The wristbands will be removed at $36 \mathrm{~h}$ after admission to ICU just after the final outcome measurement. This same procedure will be applied to participants in the placebo (sham) wristband group, with the point of difference being that they will have a sham (without bead) Seaband wristband applied to their wrists. All members of the research team will receive training and a standardised procedure manual (detailing protocol, plans for dealing with intervention fidelity issues, and monitoring the delivery and receipt of the intervention ${ }^{27}$ ), to ensure protocol consistency. All patients will receive identical information and instructions regarding the study, relayed by RRN and also in an information sheet provided at enrolment.

\section{Concurrent treatment}

Patients in both groups will have a standardised anaesthetic protocol for premedication, anaesthesia and postoperative pain and nausea management. In cardiac anaesthesia, it is very unusual to give PONV prophylaxis either in the operating theatre or in the ICU. The treatment of PONV in this population is expectant: that is, patients are treated for PONV only when they display signs/symptoms of nausea or vomiting. This is consistent with standards of care, in Australia and internationally, given that the variable time of waking and ventilator weaning of patients is often unpredictable at the time of surgical case completion in theatre. Premedication will be standardised to temazepam $10-30 \mathrm{mg} /$ diazepam $5-10 \mathrm{mg} 1 \mathrm{~h}$ prior to surgery; anaesthesia induced with midazolam $0.03-0.1 \mathrm{mg} / \mathrm{kg}$, fentanyl $5-15 \mathrm{~g} / \mathrm{kg}$, propofol $\quad 0.25-1.25 \mathrm{mg} / \mathrm{kg}$ and pancuronium $0.1 \mathrm{mg} / \mathrm{kg} /$ rocuronium $0.75-1.2 \mathrm{mg} / \mathrm{kg}$. Anaesthesia will be maintained with: propofol infusion $2-5 \mathrm{mg} / \mathrm{kg} / \mathrm{h}$, sevoflurance administered precardiopulmonary bypass for ischaemic preconditioning at discretion of attending anaesthetist, air $/ \mathrm{O}_{2}$ mix at discretion of attending anaesthetist. Transfer to ICU, patients will be maintained on propofol infusion and fentanyl infusion at $5-25 \mu \mathrm{g} / \mathrm{h}$ with no prophylactic antiemetics administered (current usual care). Participants will be sedated with the aforementioned propofol and fentanyl infusions until determined appropriate to extubation of the artificial airway, and then maintained on fentanyl via patient-controlled analgesia (background of $0-25 \mu \mathrm{g} / \mathrm{h}$; bolus of $5-25 \mu \mathrm{g}$ every $5 \mathrm{~min}$ ) for $48 \mathrm{~h}$ or until cardiac drain are removed postoperatively. A standardised rescue antiemetic protocol involving the use of a grading system will be used (see table 1). For any patient requiring nasogastric treatment postoperatively this will be recorded (given this prevents gastric distension and vomiting) and gastric volume recorded for $36 \mathrm{~h}$.

\begin{tabular}{|c|c|c|}
\hline Symptoms & $\begin{array}{l}\text { Nausea } \\
\text { score }\end{array}$ & Treatment \\
\hline None & 0 & No treatment \\
\hline Mild & $1-3$ & $\begin{array}{l}\text { Rescue antiemetic (metoclopramide } \\
10-20 \mathrm{mg} \text { ) }\end{array}$ \\
\hline Moderate & $4-6$ & $\begin{array}{l}\text { Rescue antiemetic (metoclopramide } \\
10-20 \mathrm{mg}+\text { ondansetron } 4-8 \mathrm{mg} \text { ) }\end{array}$ \\
\hline Severe & $7-10$ & $\begin{array}{l}\text { Rescue antiemetic (metoclopamide } \\
10-20 \mathrm{mg}+\text { ondansetron } 4-8 \mathrm{mg} \text { ). If } \\
\text { no response in } 30 \mathrm{~min} \text { : } \\
\text { dexamethasone } 8 \mathrm{mg} \text {, then } \\
\text { droperidol } 0.625 \mathrm{mg} \text {. Change of } \\
\text { PCA narcotic if no effect within } \\
30 \mathrm{~min} \text {. }\end{array}$ \\
\hline $\begin{array}{l}\text { Retching/ } \\
\text { vomiting }\end{array}$ & NA & As per protocol for severe nausea \\
\hline
\end{tabular}




\section{Outcome measures}

All data will be collected using structured case report forms by staff blinded to treatment groups. This method of interviewer-led self-report data collection will minimise missing data. Nausea will be assessed at six time points: $6 \mathrm{~h}$ from arrival to the ICU; $12 \mathrm{~h}$ postarrival; then four hourly up to $24 \mathrm{~h}$; and then at $36 \mathrm{~h}$ on a 10-point scale (table 1) and aggregated into 'all', 'mild', 'moderatesevere' and 'severe' nausea. All episodes of retching or vomiting in the $36 \mathrm{~h}$ time period will be recorded. Rescue antiemetic will be given and recorded for patients who experience mild-severe nausea or an episode of vomiting within the $36 \mathrm{~h}$ study period (table 1 ). Reasons for non-adherence to intervention protocol will be recorded. Time to first rescue treatment will be recorded. Reasons for attrition will be recorded. Participants will self-assess their quality of recovery on the morning of the fourth postoperative day using a 15-item questionnairethe QOR-15. ${ }^{28}$ Any adverse event (AE) from the wristbands will be assessed and recorded at each assessment time point. Postoperative AEs will also be recorded. Healthcare resource use related to the management of nausea and vomiting will be assessed and costed. This will include: band use; frequency, dose, route and duration of rescue antiemetics; length of stay in ICU and length of stay in hospital post-ICU; and costs associated with any adverse effects of the PC6 stimulation device or the antiemetics. Demographic information will be collected at preoperative/baseline. This will include participant's age, gender, and body mass index. Probability of PONV will be predicted based on patient-related factors using the Apfel risk score. ${ }^{29}$ At PC6 stimulation device removal, the RRN will ask the patient (if able) about their satisfaction with their PONV care on a 10 -point scale (' $0=$ completely dissatisfied', ' $10=$ completely satisfied'). The Study Manager will oversee data quality including undertaking periodic audits and generation of data queries for all missing or improbable values. Clinical staff will be invited to participate in either group or individual semistructured interviews about the clinical use, feasibility, acceptability and challenges in using the acupressure wristbands for PONV in clinical practice, and their trial involvement. The interview schedule will be informed by the Theoretical Domains Framework, ${ }^{30}$ which will enhance the understanding of any intervention fidelity issues identified, the perceived risks, benefits and barriers to the use of acupressure bands so that we can develop strategies to facilitate practice change at the study conclusion.

\section{Data analysis}

Data from the case report forms will be entered and analysed under the direction of a $\mathrm{PhD}$ qualified statistical epidemiologist blinded to allocation. Prior to analysis, all missing data and improbable values will be checked against source data. The primary end point will be occurrence of nausea and/or vomiting within $36 \mathrm{~h}$ of the end of surgery. Secondary end points will be nausea and vomiting separately, occurrence of early $(\leq 16 \mathrm{~h})$ and late $(>16 \mathrm{~h}$ including repeat events) PONV, QOR score, need for rescue antiemetic therapy and band-related as well as postoperative AEs. We will use $\chi^{2}$ test (or Fishers Exact test) to compare frequency of nausea and vomiting (all types, nausea, vomiting, early PONV, late PONV, moderate-severe nausea, severe nausea and need for rescue antiemetics) in the two treatment groups. QOR and number of rescue antiemetics will be compared using Wilcoxon two-sample test. Cumulative incidence of rescue antiemetic treatment over time will be plotted using the Kaplan-Meier method and compared across the two treatment groups. We will perform stepwise multiple logistic regression analyses for early and late postoperative nausea and vomiting to identify predictors of these events. The results will be presented as adjusted ORs with 95\% CIs; we will adjust for all variables that could be independently explanatory at a $\mathrm{p} \leq 0.1$ for respective end points. Analyses will be performed according to the intention-to-treat principle, although a per protocol analysis will be undertaken as a secondary analysis to consider the likely effect on outcome measure of randomised patient attrition prior to and during treatment, missing data and protocol violations. Patient survey data will be analysed to assess satisfaction with PONV care. The Theoretical Domains Framework ${ }^{30}$ will inform the analysis of the clinical staff interviews. Economic evaluation will incorporate health-related costs and assess the value for money provided by acupressure by comparing the incremental costs and effects of the intervention. Bootstrapping will be employed to compare the mean difference in the costs between groups, and to estimate a CI around the mean. ${ }^{31}$ A comparative cost-effectiveness analysis will be undertaken based on incidence of nausea or vomiting and the QOR as outcome measures. Uncertainty around incremental cost-effectiveness ratios will be tested using both one-way sensitivity analysis and non-parametric bootstrapping methods. ${ }^{31}$

\section{ETHICS AND DISSEMINATION}

Ethics approval will be sought from appropriate Human Research Ethics Committee/s (HREC) before start of the study. Participants will be supplied with detailed information regarding the study including data access, storage and confidentiality. Participants will be required to provide informed written consent and have the right of withdrawal from the study at any time. Participation burden is low, and declining to participate will have no negative effect on the patient's continued treatment at the hospitals. There are no anticipated risks to participants. Lee and Fan $^{19}$ identified that two trials in their CSR found some participants reported that wristbands were uncomfortable, and produced minor side effects. Any serious AEs will be assessed at all time points and reported to the patient's treating doctor to determine whether further diagnostic testing or treatment is warranted. All AEs will be reported in study results. Although serious AEs will be expected given the nature of the surgery, it is highly unlikely that these will be 
related to the intervention, although these will be reported to the HRECs expeditiously, with appropriate notification of the Therapeutic Goods Administration if required. Serious AEs will be monitored and reported to the HREC. Approvals for any other variations to the protocol will be sought through HREC. The acupressure bands to be used "Seaband" is a registered medical device with Australian Register of Therapeutic Goods (ARTG 109529).

\section{Data and safety monitoring plan}

A DSMC of two biostatisticians and two medically qualified researchers (independent of study) will review nausea and vomiting outcomes after $n=350$. The DSMC will check and advise whether the study needs to be stopped early (for futility) or because the intervention effect is so great that further data collection is unnecessary (with caution given the controversies over stopping early for benefit systematically overestimating treatment effects). Stopping for futility will be considered if the conditional power (the chance of detecting a statistically significant result at the end of the trial given the interim results) is very low $(\leq 15 \%) .{ }^{32}$ We will utilise a simple predefined statistical stopping rule for benefit, the Peto-Haybittle boundary, which would indicate stopping at a $\mathrm{p}$ value for treatment difference (nausea and/or vomiting) at interim analysis of $<0.001 .{ }^{33}$ However, we acknowledge that this formal rule is insufficient to prevent bias consequent on stopping early ${ }^{34}$ and we will additionally require a large number of outcome events and considerations of clinical significance over and above the statistical boundary before early stopping for benefit is contemplated. If a major postoperative complication (eg, haemorrhage requiring return to theatre, difficulty weaning from artificial ventilation or cerebrovascular event interfering with communication) is experienced, as much data as possible will be recorded to maximise the data set available for intention to treat analysis. ${ }^{35}$

\section{Reporting and dissemination}

It is anticipated that results will be well received by academic, scientific and broader communities. Dissemination will include conference presentations at national and international scientific meetings and publications in peer-reviewed journals with a high readership in anaesthetics and cardiac surgery. Study participants will receive a one-page lay-summary of results. Use of an integrated knowledge translation approach, purposely including patients and practitioners, will assist with dissemination of study findings and those involved in the study will be encouraged to participate in wider dissemination of study findings.

\section{Author affiliations}

${ }^{1}$ NHMRC Centre for Research Excellence in Nursing Interventions, Griffith Health Institute, Centre for Health Practice Innovation, Nathan, Queensland, Australia
${ }^{2}$ Department of Anaesthesia and Perfusion, Critical Care Research Group, The Prince Charles Hospital, Chermside, Queensland, Australia

${ }^{3}$ Critical Care Research Group, The Prince Charles Hospital and University of Queensland, Chermside, Queensland, Australia

${ }^{4}$ Griffith Health Institute, Centre for Health Practice Innovation and Gold Coast University Hospital, Gold Coast, Queensland, Australia

${ }^{5}$ Griffith Health Institute, Population and Social Health Research Program, Brisbane, Queensland, Australia

${ }^{6}$ Department of Clinical Epidemiology Unit, School of Population Health,

University of Queensland, Herston, Queensland, Australia

${ }^{7}$ Microbiology and Immunology Research Group, Griffith University, Gold Coast, Queensland, Australia

${ }^{8}$ Critical Care Research Group, The Prince Charles Hospital, Chermside, Queensland, Australia

Contributors The protocol was written by $\mathrm{MC}$ and was critically reviewed by CR, IR, KS, APM, TC, SD and AS. All authors made a significant contribution to the conception and design of the study protocol. All authors gave approval for the publication.

Competing interests None.

Provenance and peer review Not commissioned; externally peer reviewed.

Open Access This is an Open Access article distributed in accordance with the Creative Commons Attribution Non Commercial (CC BY-NC 4.0) license, which permits others to distribute, remix, adapt, build upon this work noncommercially, and license their derivative works on different terms, provided the original work is properly cited and the use is non-commercial. See: http:// creativecommons.org/licenses/by-nc/4.0/

\section{REFERENCES}

1. Carlise J, Stevenson C. Drugs for preventing postoperative nausea and vomiting. Cochrane Database Syst Rev 2006;(3):CD004125.

2. AlHW. Australian hospital statistics 2011-2012. Canberra: AlHW, 2013. Contract No.: Health services series no. 50. Cat. no. HSE 134

3. AlHW. Australia's health 2012. Canberra: AlHW, 2012. Contract No.: Australia's health series no.13. Cat. no. AUS 156

4. British Heart Foundation. Coronary heart disease statistics: a compendium of health statics, 12th edn. London: British Health Foundation, 2012:204. http://www.bhf.org.uk/plugins/Publications SearchResults/DownloadFile. aspx?docid=508b8b91-13014ad7-bc7e-7f413877548b\&version $=-1 \&$ title $=$ Coronary+Heart +Disease+Statistics+2012+\& resource=G608\%2f1012\%2fCHA

5. Checketts M, Gilhooly C, Kenny G. Patient-maintained analgesia with target-controlled alfentanil infusion after cardiac surgery: a comparison with morphine PCA. Br J Anaesth 1998;80:748-51.

6. Grebenik C, Allman C. Nausea and vomiting after cardiac surgery. Br J Anaesth 1996;77:356-9.

7. Woodward D, Sherry K, Harrison D. Antiemetic prophylaxis in cardiac surgery: comparison of metoclopramide and ondansetron. Br J Anaesth 1999;83:933-5.

8. Korinenko Y, Vincent A, Cutshall S, et al. Efficacy of acupuncture in prevention of postoperative nausea in cardiac surgery patients. Ann Thorac Surg 2009;88:537-42.

9. Bainbridge D, Martin J, Cheng D. Patient-controlled versus nurse-controlled analgesia after cardiac surgery-a meta-analysis. Can J Anaesth 2006;53:492-9.

10. Klein A, Djaiani G, Karshi J, et al. Acupressure wristbands for the prevention of postoperative nausea and vomiting in adults undergoing cardiac surgery. J Cardiothorac Vasc Anesth 2004;18:68-71.

11. Lee A, Gin T, Lau A, et al. A comparison of patients and health care professionals' preferences for symptoms during immediate postoperative recovery and the management of postoperative nausea and vomiting. Anesth Analg 2005;100:87-93.

12. Gan T. Risk factors for postoperative nausea and vomiting. Anesth Analg 2006;102:1884-98.

13. Myles $P$, Williams $D$, Hendrata $M$, et al. Patient satisfaction after anaesthesia and surgery: results of a prospective survey of 10,811 patients. Br J Anaesth 2000;84:6-10.

14. WHO Western Pacific Region Office. Guidelines for clinical research on acupuncture. Manila, Philippines: WHO, 1995.

15. Abraham J. Acupressure and acupuncture in preventing and managing postoperative nausea and vomiting in adults. J Perioper Pract 2008;18:543-51. 
16. Turgut S, Ozalp G, Dikmen S, et al. Acupressure for postoperative nausea and vomiting in gynaecological patients receiving patient-controlled analgesia. Eur J Anaesthesiol 2007; 24:87-91.

17. Sadighha A, Nurai N. Acupressure wristbands versus metoclopramide for the prevention of postoperative nausea and vomiting. Ann Saudi Med 2008;28:287-91.

18. WHO Western Pacific Region Office. WHO standard acupuncture point locations in the Western Pacific Region. Manila, Philippines: WHO, 2008.

19. Lee A, Fan L. Stimulation of the wrist acupuncture point $P 6$ for preventing postoperative nausea and vomiting. Cochrane Database Syst Rev 2009;(2):CD003281.

20. Medical Research Council. A framework for development and evaluation of RCTs for complex interventions to improve health. London: MRC, 2000.

21. Lewin S, Glenton C, Oxman A. Use of qualitative methods alongside randomised controlled trials of complex healthcare interventions: methodological study. BMJ 2009;339:b3496.

22. Moher D, Hopewell S, Schulz K, et al. CONSORT 2010 explanation and elaboration: updated guidelines for reporting parallel group randomised trials. BMJ 2010;340:c869.

23. MacPherson $\mathrm{H}$, Altman $\mathrm{D}$, Hammerschlag $\mathrm{R}$, et al. Revised STandards for Reporting Interventions in Clinical Trials of Acupuncture (STRICTA): extending the CONSORT Statement. $J$ Evid Based Med 2010;3:140-55.

24. Chinese University of Hong Kong. Center for Clinical Trials calculator. http://www.cct.cuhk.edu.hk/stat/proportion/tspp_sup.htm

25. Apfel C, Roewer N. Risk assessment of postoperative nausea and vomiting. Int Anesth Clin 2003;41:13-32.
26. Higgins J, Altman D, Sterne J.; on behalf of the Cochrane Statistical Methods Group and the Cochrane Bias Methods Group, The Cochrane Collaboration. Assessing risk of bias in included studies. The Cochrane Collection, 2011.

27. Spillane $\mathrm{V}$, Byrne $\mathrm{M}, \mathrm{Byrne} \mathrm{M}$, et al. Monitoring treatment fidelity in a randomized controlled trial of a complex intervention. J Adv Nurs 2007;60:343-52.

28. Stark P, Myles P, Burke J. Development of psychometric evaluation of a postoperative quality of recovery score. Anesthesiology 2013;118:1332-40.

29. Apfel C, Breim C, Haubitz I, et al. A risk score to predict the probability of postoperative vomiting in adults. Acta Anaesthesiol Scand 1998;42:495-501.

30. Michie S, Johnston M, Abraham C, et al. Making psychological theory useful for implementing evidence based practice: a consensus approach. Qual Saf Health Care 2005;14:26-33.

31. Briggs $A H$, Wonderling DE, Mooney CZ. Pulling cost-effectiveness analysis up by its bootstraps: a non-parametric approach to confidence interval estimation. Health Econ 1997;4:327-40.

32. Jitlal M, Khan L, Lee SM, et al. Stopping clinical trials early for futility: retrospective analysis of several randomised clinical studies. Br J Cancer 2012;107:910-17.

33. Pocock SJ. When (not) to stop a clinical trial for benefit. JAMA 2005;294:2228-30.

34. Bassler D, Briel M, Montori VM, et al. Stopping randomized trials early for benefit and estimation of treatment effects. JAMA 2010;303:1180-7.

35. Polit D, Gillespie B. Intention-to-treat in randomized controlled trials: recommendations for a total trial strategy. Res Nurs Health 2010;33:355-68. 